\title{
SLOW MANIFOLDS FOR MULTI-TIME-SCALE STOCHASTIC EVOLUTIONARY SYSTEMS*
}

\author{
HONGBO $\mathrm{FU}^{\dagger}$, XIANMING LIU ${ }^{\ddagger}$, AND JINQIAO DUAN $\S$
}

\begin{abstract}
This article deals with invariant manifolds for infinite dimensional random dynamical systems with different time scales. Such a random system is generated by a coupled system of fastslow stochastic evolutionary equations. Under suitable conditions, it is proved that an exponentially tracking random invariant manifold exists, eliminating the fast motion for this coupled system. It is further shown that if the scaling parameter tends to zero, the invariant manifold tends to a slow manifold which captures long time dynamics. For illustration, the results are applied to a few systems of coupled parabolic-hyperbolic partial differential equations, coupled parabolic partial differentialordinary differential equations, and coupled hyperbolic-hyperbolic partial differential equations.
\end{abstract}

Key words. Stochastic partial differential equations (SPDEs), random dynamical systems, multiscale systems, random invariant manifolds, slow manifolds, exponential tracking property.

Mathematics Subject Classifications (2010). 60H15, 70K70, 37L55, 37D10.

\section{Introduction}

The theory of invariant manifolds serves as a tool for analyzing dynamical behaviors of deterministic systems. It was first introduced by Hadamard [20], then by Lyapunov [24] and Perron [29] for deterministic systems. It has been further developed by many authors for infinite dimensional deterministic systems; see, e.g., $[30,2,9,12,21]$. More recently, invariant manifolds have been investigated for infinite dimensional stochastic systems; see [17, 18, 23, 25, 3, 6, 15, 14] among others.

Some systems evolve on fast and slow time scales, and may thus be modeled by coupled singularly perturbed stochastic ordinary or partial differential equations (SDEs or SPDEs). For SDEs with two time scales, Schmalfuß and Schneider [32] have recently investigated random inertial manifolds that eliminate the fast variables by a fixed point technique based on a random graph transformation. They show that the inertial manifold tends to another so-called slow manifold as the scaling parameter goes to zero. Qualitative analysis for the behavior of the slow manifold for slow-fast SDEs on the long time scales can be found in Wang and Roberts [34].

In the present paper, we consider invariant manifolds for stochastic fast-slow systems in infinite dimension. Namely we investigate the following system of fastslow stochastic evolutionary equations, which could be coupled SPDEs, or coupled E.

*Received: July 16, 2011; accepted (in revised form): April 30, 2012. Communicated by Weinan

This work was supported by the NSFC grants $(10901065,10971225,11028102)$, the Chinese Universities' Basic Research Fund, the NSF Grant 1025422, the open funding of LNM at the Institute of Mechanics of Chinese Academy of Science, the Fundamental Research Funds for the Central Universities (HUST No.2010ZD037 and No. 2011QNQ170), and the Research Fund for the Doctoral Program of Higher Education of China (20110142120036).

${ }^{\dagger}$ College of Mathematics and Computer Science, Wuhan Textile University, Wuhan 430073, China (hbfuhust@gmail.com).

${ }^{\ddagger}$ School of Mathematics and Statistics, Huazhong University of Science and Technology, Wuhan 430074, China (mathliuxm@yahoo.cn).

$\S$ Institute for Pure and Applied Mathematics, University of California, Los Angeles, CA 90095 and Department of Applied Mathematics Illinois Institute of Technology, Chicago, IL 60616, USA (duan@iit.edu). 
SPDEs-SDEs,

$$
\begin{aligned}
& \dot{x}^{\epsilon}=\frac{1}{\epsilon} A x^{\epsilon}+\frac{1}{\epsilon} f\left(x^{\epsilon}, y^{\epsilon}\right)+\frac{\sigma}{\sqrt{\epsilon}} \dot{w}, \quad \text { in } \mathrm{H}_{1}, \\
& \dot{y}^{\epsilon}=B y^{\epsilon}+g\left(x^{\epsilon}, y^{\epsilon}\right), \quad \text { in } \mathrm{H}_{2},
\end{aligned}
$$

where $A$ and $B$ are generators of $C_{0}$-semigroups, and the interaction functions $f$ and $g$ are continuous. The noise process $w=\sum_{j=1}^{m} h_{j} w_{j}$, where $\left\{w_{j}\right\}_{j=1}^{m}$ are two-sided Wiener processes (or Brownian motions) taking values in $\mathbb{R}$ and $h_{j}(1 \leq j \leq m)$ are given elements in $H_{1}$. The small parameter $\epsilon>0$ represents the ratio of the two time scales. The precise conditions on these quantities will be given in Section 3, and allow our framework to deal with multiscale coupled parabolic-hyperbolic systems and coupled hyperbolic-hyperbolic systems.

It is worthy mentioning that in the situation we consider here, the noise perturbation of the fast motion equation is of additive type. The reason is that the problem for existence of random dynamical systems generated by stochastic partial differential equations with general multiplicative noise is still unsolved (for details see [17]). The main goal in this paper is to establish, for $\epsilon>0$ small enough, the existence of a random invariant manifold $M^{\epsilon}$ with an exponential tracking property for the above stochastic system. Thus as a consequence, this system can be reduced to an evolutionary equation with a modified nonlinear term, which is useful for describing the long time behavior of the original coupled stochastic system. There are usually two approaches to construction of invariant manifolds: the Hadamard graph transform method (see [31, 17]) and the Lyapunov-Perron method (see $[11,18,6])$. We achieve our results by the latter, which is different from the method of random graph transformation in [32]. In this approach one key assumption is that the Lipschitz constant of the nonlinear term in the fast component is small enough compared to the decay rate of the linear operator $A$. In particular, under suitable conditions it is further shown that this manifold $M^{\epsilon}$ can be asymptotically approximated for $\epsilon$ sufficiently small by a slow manifold $M^{0}$ for a reduced stochastic system. We note that, in the case of the Lyapunov-Perron method applied to a coupled stochastic systems, the existence of a random invariant manifold for the coupled stochastic parabolic-hyperbolic equations, that do not contain two widely separated characteristic timescales, is obtained by Caraballo Chueshov and Langa in [6]. We also remark that, whereas the existence of a slow manifold is not studied, in their paper the authors also verify that this random manifold converges to its deterministic counterpart when the intensity of noise tends to zero.

This paper is organized as follows. In Section 2, some basic concepts in random dynamical systems and random invariant manifolds are recalled. Our framework is presented in Section 3. In Section 4, we establish the existence of a random invariant manifold $M^{\epsilon}$ possessing an exponential tracking property, and then in Section 5 we show that $M^{\epsilon}$ converges to a slow manifold $M^{0}$ with rate of order 1 . Section 6 is devoted to a few illustrative examples. Remarks on local manifolds for systems with local Lipschitz nonlinearities are given in Section 7.

\section{Preliminaries on random dynamical systems}

We now recall basic concepts in random dynamical systems (RDS) and random invariant manifolds (RIM). For more details, see [1, 17, 18]. 
Definition 2.1. Let $(\Omega, \mathscr{F}, \mathbb{P})$ be a probability space and $\theta=\left\{\theta_{t}\right\}_{t \in \mathbb{R}}$ be a flow on $\Omega$ which is defined as a mapping

$$
\theta: \mathbb{R} \times \Omega \mapsto \Omega
$$

which satisfies

- $\theta_{0}=i d_{\Omega}$,

- $\theta_{s} \theta_{t}=\theta_{s+t}$ for all $s, t \in \mathbb{R}$,

- the mapping $(t, \omega) \mapsto \theta_{t} \omega$ is $(\mathscr{B}(\mathbb{R}) \times \mathscr{F}, \mathscr{F})$-measurable and $\theta_{t} \mathbb{P}=\mathbb{P}$ for all $t \in \mathbb{R}$. Then the quadruple $(\Omega, \mathcal{F}, \mathbb{P}, \theta)$ is called a driving dynamical system.

We will work on the driving dynamical system represented by a Wiener process. To be more precise, let $\Omega=C_{0}\left(\mathbb{R}, \mathbb{R}^{m}\right)$ be the continuous paths $\omega(t)$ on $\mathbb{R}$ with values $\mathbb{R}^{m}$ such that $\omega(0)=0$. This set is equipped with the compact-open topology. Let $\mathcal{F}$ be the associated Borel $\sigma$-field and $\mathbb{P}$ be the Wiener measure. Then we identify $\omega$ with

$$
\left(w_{1}(t), w_{2}(t), \cdots, w_{m}(t)\right)=\omega(t), t \in \mathbb{R} .
$$

The operators $\theta_{t}$ forming the flow are given by the Wiener shift:

$$
\theta_{t} \omega(\cdot)=\omega(\cdot+t)-\omega(t), \omega \in \Omega, t \in \mathbb{R} .
$$

Note that the measure $\mathbb{P}$ is invariant with respect to the above flow, and therefore the quadruple $(\Omega, \mathcal{F}, \mathbb{P}, \theta)$ is a driving dynamical system.

Definition 2.2. Let $\left(\mathbb{H}, d_{\mathbb{H}}\right)$ be a metric space with Borel $\sigma-$ field $\mathscr{B}(\mathbb{H})$. A cocycle is a $\left(\mathscr{B}\left(\mathbb{R}^{+}\right) \times \mathscr{F} \times \mathscr{B}(\mathbb{H}), \mathscr{B}(\mathbb{H})\right.$ )- measurable mapping

$$
\phi: \mathbb{R}^{+} \times \Omega \times \mathbb{H} \mapsto \mathbb{H}
$$

such that

$$
\begin{aligned}
& \phi(0, \omega, x)=x, \\
& \phi(t+s, \omega, x)=\phi\left(t, \theta_{s} \omega, \phi(s, \omega, x)\right),
\end{aligned}
$$

for $t, s \in \mathbb{R}^{+}, \omega \in \Omega$, and $x \in \mathbb{H}$. Then $\phi$ together with the driving system $\theta$ forms a random dynamical system (RDS).

A RDS is called continuous (differentiable) if $x \rightarrow \phi(t, \omega, x)$ is continuous (differentiable) for $t \geq 0$ and $\omega \in \Omega$. A family of nonempty closed sets $M=\{M(\omega)\}$ contained in a metric space $\left(\mathbb{H},\|\cdot\|_{\mathbb{H}}\right)$ is called a random set if for every $y \in \mathbb{H}$ the mapping

$$
\omega \rightarrow \inf _{x \in M(\omega)}\|x-y\|_{\mathbb{H}}
$$

is a random variable. Now we introduce the random invariant manifold concept.

Definition 2.3. A random set $M(\omega)$ is called a positively invariant set if

$$
\phi(t, \omega, M(\omega)) \subset M\left(\theta_{t} \omega\right), \text { for } t \geq 0, \omega \in \Omega .
$$

If $M$ can be represented as a graph of a Lipschitz mapping

$$
\psi(\cdot, \omega): H_{1} \rightarrow H_{2}, \mathbb{H}=H_{1} \times H_{2}
$$


such that

$$
M(\omega)=\left\{\left(x_{1}, \psi\left(x_{1}, \omega\right)\right): x_{1} \in H_{1}\right\}
$$

then $M(\omega)$ is called a Lipschitz random invariant manifold. If, in addition, for every $x \in \mathbb{H}$, there exists an $x^{\prime} \in M(\omega)$ such that for all $\omega \in \Omega$,

$$
\left\|\phi(t, \omega, x)-\phi\left(t, \omega, x^{\prime}\right)\right\|_{\mathbb{H}} \leq c_{1}\left(x, x^{\prime}, \omega\right) e^{-c_{2} t}\left\|x-x^{\prime}\right\|_{\mathbb{H}}, t \geq 0,
$$

where $c_{1}$ is a positive random variable depending on $x$ and $x^{\prime}$, while $c_{2}$ is a positive constant, then $M(\omega)$ is said to have an exponential tracking property.

\section{Framework}

Consider the following system of stochastic evolutionary equations with two time scales:

$$
\begin{aligned}
& \dot{x}^{\epsilon}=\frac{1}{\epsilon} A x^{\epsilon}+\frac{1}{\epsilon} f\left(x^{\epsilon}, y^{\epsilon}\right)+\frac{\sigma}{\sqrt{\epsilon}} \dot{w}, \quad \text { in } \mathrm{H}_{1}, \\
& \dot{y}^{\epsilon}=B y^{\epsilon}+g\left(x^{\epsilon}, y^{\epsilon}\right), \quad \text { in } \mathrm{H}_{2},
\end{aligned}
$$

where $A$ is a generator of a $C_{0}$-semigroup on the separable Hilbert space $H_{1}$, and $B$ is a generator of a $C_{0}$-group on the separable Hilbert $H_{2}$. Nonlinearities $f$ and $g$ are continuous functions,

$$
f: H_{1} \times H_{2} \mapsto H_{1}, \quad g: H_{1} \times H_{2} \mapsto H_{2},
$$

with $f(0,0)=g(0,0)=0$. The noise process $w=\sum_{j=1}^{m} h_{j} w_{j}$, where $\left\{w_{j}\right\}_{j=1}^{m}$ are twosided Wiener processes taking values in $\mathbb{R}$ and $h_{j}(1 \leq j \leq m)$ are given elements in $H_{1}$. Moreover, $\sigma$ is a nonzero constant (noise intensity), and $\epsilon$ is a small positive parameter representing the ratio of time scales in this fast-slow system. In this setting, $x^{\epsilon}$ is referred to as the "fast" component while $y^{\epsilon}$ is the "slow" component.

Denote by $\|\cdot\|_{1}$ and $\|\cdot\|_{2}$ the norms in $H_{1}$ and $H_{2}$, respectively. The norm in $\mathbb{H}=H_{1} \times H_{2}$ is denoted as $\|\cdot\|$. For the linear operators $A$ and $B$ we assume the following conditions:

(A1) Let $A$ be the generator of a $C_{0}-$ semigroup $e^{A t}$ on $H_{1}$ satisfying

$$
\left\|e^{A t} x\right\|_{1} \leq e^{-\gamma_{1} t}\|x\|_{1}, \quad t \geq 0,
$$

for all $x \in H_{1}$, with a constant (i.e., decay rate) $\gamma_{1}>0$. Moreover, $B$ is the generator of a $C_{0}$-group $e^{B t}$ on $\mathrm{H}_{2}$ satisfying

$$
\left\|e^{B t} y\right\|_{2} \leq e^{-\gamma_{2} t}\|y\|_{2}, \quad t \leq 0
$$

for all $y \in H_{2}$, with a constant $\gamma_{2} \geq 0$.

We also make the following two assumptions:

(A2) Lipschitz condition: There exists a positive constant $K$ such that for all $\left(x_{i}, y_{i}\right) \in H_{1} \times H_{2}$,

$$
\left\|f\left(x_{1}, y_{1}\right)-f\left(x_{2}, y_{2}\right)\right\|_{1} \leq K\left(\left\|x_{1}-x_{2}\right\|_{1}+\left\|y_{1}-y_{2}\right\|_{2}\right)
$$

and

$$
\left\|g\left(x_{1}, y_{1}\right)-g\left(x_{2}, y_{2}\right)\right\|_{2} \leq K\left(\left\|x_{1}-x_{2}\right\|_{1}+\left\|y_{1}-y_{2}\right\|_{2}\right) .
$$


(A3) Assume that the Lipschitz constant $K$ of the nonlinear terms in system (3.1)-(3.2) is smaller than the decay rate $\gamma_{1}$ of $A$, that is,

$$
K<\gamma_{1}
$$

REMARK 3.1. We note that the system (3.1)-(3.2) is an abstract model for various complex systems under random influences, which can be a finite-dimensional, stochastic slow-fast system analyzed in [32, 34].

Now, as in [17], we verify that the stochastic evolutionary system (3.1)-(3.2) can be transformed into a random evolutionary system which generates a RDS. For this purpose, let $\eta^{\frac{1}{\epsilon}}$ be a stationary solution of the linear stochastic evolutionary equation

$$
d \eta^{\frac{1}{\epsilon}}(t)=\frac{1}{\epsilon} A \eta^{\frac{1}{\epsilon}}(t) d t+\frac{\sigma}{\sqrt{\epsilon}} d w(t) .
$$

This means that the random variable $\eta^{\frac{1}{\epsilon}}$ with values in $H_{1}$ is defined on a $\left\{\theta_{t}\right\}_{t \in \mathbb{R}}$-invariant set of full measure such that

$$
t \rightarrow \eta^{\frac{1}{\epsilon}}\left(\theta_{t} \omega\right)
$$

is a solution version for (3.3). Let $\xi$ be the stationary solution of the linear stochastic evolutionary equation

$$
d \xi(t)=A \xi(t)+\sigma d w(t) .
$$

Then by the scale property of Wiener process, $\eta^{\frac{1}{\epsilon}}\left(\theta_{t} \omega\right)$ has the same distribution of $\xi\left(\theta_{\frac{t}{\epsilon}} \omega\right)$; see the Lemma 3.2 in [32]. For the existence of stationary solutions to stochastic evolutionary equations; see [5].

Define $X^{\epsilon}=x^{\epsilon}-\eta^{\frac{1}{\epsilon}}\left(\theta_{t} \omega\right)$ and $Y^{\epsilon}=y^{\epsilon}$. Then the original evolutionary system (3.1)-(3.2) is converted to the following random evolutionary system:

$$
\begin{aligned}
& d X^{\epsilon}=\frac{1}{\epsilon} A X^{\epsilon} d t+\frac{1}{\epsilon} F\left(X^{\epsilon}, Y^{\epsilon}, \theta_{t}^{\epsilon} \omega\right) d t \\
& d Y^{\epsilon}=B Y^{\epsilon} d t+G\left(X^{\epsilon}, Y^{\epsilon}, \theta_{t}^{\epsilon} \omega\right) d t
\end{aligned}
$$

where

$$
\begin{aligned}
& F\left(X^{\epsilon}, Y^{\epsilon}, \theta_{t}^{\epsilon} \omega\right)=f\left(X^{\epsilon}+\eta^{\frac{1}{\epsilon}}\left(\theta_{t} \omega\right), Y^{\epsilon}\right), \\
& G\left(X^{\epsilon}, Y^{\epsilon}, \theta_{t}^{\epsilon} \omega\right)=g\left(X^{\epsilon}+\eta^{\frac{1}{\epsilon}}\left(\theta_{t} \omega\right), Y^{\epsilon}\right) .
\end{aligned}
$$

Let $Z^{\epsilon}\left(t, \omega, Z_{0}\right)=\left(X^{\epsilon}\left(t, \omega, X_{0}, Y_{0}\right), Y^{\epsilon}\left(t, \omega, X_{0}, Y_{0}\right)\right)$ be the solution of (3.4)-(3.5) with initial data $\left(X^{\epsilon}(0), Y^{\epsilon}(0)\right)=\left(X_{0}, Y_{0}\right):=Z_{0}$. Then the solution operator of (3.4)$(3.5)$,

$$
\Phi^{\epsilon}\left(t, \omega,\left(X_{0}, Y_{0}\right)\right)=\left(X^{\epsilon}\left(t, \omega, X_{0}, Y_{0}\right), Y^{\epsilon}\left(t, \omega, X_{0}, Y_{0}\right)\right),
$$

defines a random dynamical system [17]. Furthermore,

$$
\phi^{\epsilon}(t, \omega):=\Phi^{\epsilon}(t, \omega)+\left(\eta^{\frac{1}{\epsilon}}\left(\theta_{t} \omega\right), 0\right), t \geq 0, \omega \in \Omega
$$

is the random dynamical system generated by the original system (3.1)-(3.2). 
We introduce some notations. Let $\mu$ be a positive number satisfying

$$
\gamma_{1}-\mu>K
$$

For any $\alpha \in \mathbb{R}$, define Banach spaces

$$
C_{\alpha}^{i,-}=\left\{\varphi:(-\infty, 0] \mapsto H_{i} \text { is continuous and } \sup _{t \leq 0}\left\|e^{-\alpha t} \varphi(t)\right\|_{i}<\infty\right\}
$$

with the norm $\|\varphi\|_{C_{\alpha}^{i,-}}=\sup _{t \leq 0}\left\|e^{-\alpha t} \varphi(t)\right\|_{i}$ for $i=1,2$. Similarly, we define Banach spaces

$$
C_{\alpha}^{i,+}=\left\{\varphi:[0, \infty,) \mapsto H_{i} \text { is continuous and } \sup _{t \geq 0}\left\|e^{-\alpha t} \varphi(t)\right\|_{i}<\infty\right\}
$$

with the norm $\|\varphi\|_{C_{\alpha}^{i,+}}=\sup _{t \geq 0}\left\|e^{-\alpha t} \varphi(t)\right\|_{i}$ for $i=1,2$. Let $C_{\alpha}^{ \pm}$be the product Banach spaces $C_{\alpha}^{ \pm}:=C_{\alpha}^{1, \pm} \times C_{\alpha}^{2, \pm}$, with the norm

$$
\|z\|_{C_{\alpha}^{ \pm}}=\|x\|_{C_{\alpha}^{1, \pm}}+\|y\|_{C_{\alpha}^{2, \pm}}, z=(x, y) \in C_{\alpha}^{ \pm} .
$$

\section{Exponentially tracking invariant manifolds}

In this section, we prove the existence of a Lipschitz continuous invariant manifold $M^{\epsilon}(\omega)$, with an exponential tracking property, for the random evolutionary system $(3.4)-(3.5)$.

Define

$$
M^{\epsilon}(\omega) \triangleq\left\{Z_{0} \in \mathbb{H}: Z^{\epsilon}\left(\cdot, \omega, Z_{0}\right) \in C_{-\frac{\mu}{\epsilon}}^{-}\right\} .
$$

This is the set of all initial data through which solutions are bounded by $e^{-\frac{\mu}{\epsilon} t}$. We shall use Lyapunov-Perron method to prove that $M^{\epsilon}(\omega)$ is an invariant manifold described by the graph of a Lipschitz function. For this we will need the following properties of the random function $Z^{\epsilon}\left(\cdot, \omega, Z_{0}\right)$ (see [18]).

Lemma 4.1. Suppose that $Z^{\epsilon}(\cdot, \omega)=\left(X^{\epsilon}(\cdot, \omega), Y^{\epsilon}(\cdot, \omega)\right)$ is in $C_{-\frac{\mu}{\epsilon}}^{-}$. Then $Z^{\epsilon}(t, \omega)$ is the solution of (3.4)-(3.5) with initial data $Z_{0}=\left(X_{0}, Y_{0}\right)$ if and only if $Z^{\epsilon}(\cdot, \omega)$ satisfies

$$
\left(\begin{array}{c}
X^{\epsilon}(t) \\
Y^{\epsilon}(t)
\end{array}\right)=\left(\begin{array}{c}
\frac{1}{\epsilon} \int_{-\infty}^{t} e^{\frac{A(t-s)}{\epsilon}} F\left(X^{\epsilon}(s), Y^{\epsilon}(s), \theta_{s}^{\epsilon} \omega\right) d s \\
e^{B t} Y_{0}+\int_{0}^{t} e^{B(t-s)} G\left(X^{\epsilon}(s), Y^{\epsilon}(s), \theta_{s}^{\epsilon} \omega\right) d s
\end{array}\right) .
$$

THEOREM 4.2 (Invariant manifolds). Assume that (A1)-(A3) hold and that $\epsilon>0$ is sufficiently small. Then the random dynamical system defined by (3.4)-(3.5) has a Lipschitz random invariant manifold $M^{\epsilon}(\omega)$ represented as a graph

$$
M^{\epsilon}(\omega)=\left\{\left(H^{\epsilon}\left(\omega, Y_{0}\right), Y_{0}\right): Y_{0} \in H_{2}\right\}
$$

where

$$
H^{\epsilon}(\cdot, \cdot): \Omega \times H_{2} \mapsto H_{1}
$$

is the graph mapping with Lipschitz constant satisfying

$$
\operatorname{LipH}^{\epsilon}(\omega, \cdot) \leq \frac{K}{\left(\gamma_{1}-\mu\right)\left[1-K\left(\frac{1}{\gamma_{1}-\mu}+\frac{\epsilon}{\mu-\epsilon \gamma_{2}}\right)\right]}, \omega \in \Omega
$$


Proof. We adapt the method of Lyapunov-Perron to fast-slow random dynamical systems. To construct an invariant manifold for system (3.4)-(3.5) we first consider integral equations

$$
\left(\begin{array}{c}
X^{\epsilon}(t) \\
Y^{\epsilon}(t)
\end{array}\right)=\left(\begin{array}{c}
\frac{1}{\epsilon} \int_{-\infty}^{t} e^{\frac{A(t-s)}{\epsilon}} F\left(X^{\epsilon}(s), Y^{\epsilon}(s), \theta_{s}^{\epsilon} \omega\right) d s \\
e^{B t} Y_{0}+\int_{0}^{t} e^{B(t-s)} G\left(X^{\epsilon}(s), Y^{\epsilon}(s), \theta_{s}^{\epsilon} \omega\right) d s
\end{array}\right), t \leq 0
$$

A solution of (4.1) is denoted by $Z^{\epsilon}\left(t, \omega, Z_{0}\right)=\left(X^{\epsilon}\left(t, \omega, Y_{0}\right), Y^{\epsilon}\left(t, \omega, Y_{0}\right)\right)$. Introduce the operators $\mathcal{J}_{1}^{\epsilon}: C_{-\frac{\mu}{\epsilon}}^{-} \mapsto C_{-\frac{\mu}{\epsilon}}^{1,-}$ and $\mathcal{J}_{2}^{\epsilon}: C_{-\frac{\mu}{\epsilon}}^{-} \mapsto C_{-\frac{\mu}{\epsilon}}^{2,-}$ by means of

$$
\begin{gathered}
\mathcal{J}_{1}^{\epsilon}(z(\cdot))[t]=\frac{1}{\epsilon} \int_{-\infty}^{t} e^{\frac{A(t-s)}{\epsilon}} F\left(x(s), y(s), \theta_{s}^{\epsilon} \omega\right) d s, \\
\mathcal{J}_{2}^{\epsilon}(z(\cdot))[t]=e^{B t} Y_{0}+\int_{0}^{t} e^{B(t-s)} G\left(x(s), y(s), \theta_{s}^{\epsilon} \omega\right) d s,
\end{gathered}
$$

for $t \leq 0$, and define the mapping $\mathcal{J}^{\epsilon}$ by

$$
\mathcal{J}^{\epsilon}(z(\cdot)):=\left(\begin{array}{c}
\mathcal{J}_{1}^{\epsilon}(z(\cdot)) \\
\mathcal{J}_{2}^{\epsilon}(z(\cdot))
\end{array}\right) .
$$

It can be verified that $\mathcal{J}^{\epsilon}$ maps $C_{-\frac{\mu}{\epsilon}}^{-}$into itself. To this end, taking $z=(x, y) \in C_{-\frac{\mu}{\epsilon}}^{-}$, we have that

$$
\begin{aligned}
\left\|\mathcal{J}_{1}^{\epsilon}(z)\right\|_{C_{-\frac{\mu}{\epsilon}}^{1,-}} & \leq \frac{K}{\epsilon} \sup _{t \leq 0}\left\{e^{\frac{\mu}{\epsilon} t} \int_{-\infty}^{t} e^{\frac{-\gamma_{1}(t-s)}{\epsilon}}\left(\|x(s)\|_{1}+\|y(s)\|_{2}\right) d s\right\} \\
& \leq \frac{K}{\epsilon} \sup _{t \leq 0}\left\{\int_{-\infty}^{t} e^{\left(\frac{-\gamma_{1}}{\epsilon}+\frac{\mu}{\epsilon}\right)(t-s)} d s\right\}\|z\|_{C_{-\frac{\mu}{\epsilon}}^{-}} \\
& =\frac{K}{\gamma_{1}-\mu}\|z\|_{C_{-\frac{\mu}{\epsilon}}^{-}}
\end{aligned}
$$

and

$$
\begin{aligned}
\left\|\mathcal{J}_{2}^{\epsilon}(z)\right\|_{C_{-\frac{\mu}{\epsilon}}^{2,-}} & \leq K \sup _{t \leq 0}\left\{e^{\frac{\mu}{\epsilon} t} \int_{t}^{0} e^{-\gamma_{2}(t-s)} e^{-\frac{\mu}{\epsilon} s} d s\right\}\|z\|_{C_{-\frac{\mu}{\epsilon}}^{-}} \\
& \leq K \sup _{t \leq 0}\left\{\int_{t}^{0} e^{\left(-\gamma_{2}+\frac{\mu}{\epsilon}\right)(t-s)} d s\right\}\|z\|_{C_{-\frac{\mu}{\epsilon}}^{-}} \\
& =\frac{\epsilon K}{\mu-\epsilon \gamma_{2}}\|z\|_{C_{-\frac{\mu}{\epsilon}}^{-}} .
\end{aligned}
$$

Hence, by definition of $\mathcal{J}^{\epsilon}$ we obtain

$$
\left\|\mathcal{J}^{\epsilon}(z)\right\|_{C_{-\frac{\mu}{\epsilon}}^{-}} \leq \kappa\left(K, \gamma_{1}, \gamma_{2}, \mu, \epsilon\right)\|z\|_{C_{-\frac{\mu}{\epsilon}}^{-}}
$$

with 


$$
\kappa\left(K, \gamma_{1}, \gamma_{2}, \mu, \epsilon\right)=\frac{K}{\gamma_{1}-\mu}+\frac{\epsilon K}{\mu-\epsilon \gamma_{2}} .
$$

Thus, we conclude that $\mathcal{J}^{\epsilon}$ maps $C_{-\frac{\mu}{\epsilon}}^{-}$into itself.

Next we show that the mapping $\mathcal{J}^{\epsilon}$ is contractive. To this end, taking $z=$ $(x, y), \bar{z}=(\bar{x}, \bar{y}) \in C_{-\frac{\mu}{\epsilon}}^{-}$, we have that

$$
\begin{aligned}
\left\|\mathcal{J}_{1}^{\epsilon}(z)-\mathcal{J}_{1}^{\epsilon}(\bar{z})\right\|_{C_{-\frac{\mu}{\epsilon}}^{1,-}} \leq & \frac{K}{\epsilon} \sup _{t \leq 0}\left\{e ^ { \frac { \mu } { \epsilon } t } \int _ { - \infty } ^ { t } e ^ { \frac { - \gamma _ { 1 } ( t - s ) } { \epsilon } } \left(\|x(s)-\bar{x}(s)\|_{1}\right.\right. \\
& \left.\left.+\|y(s)-\bar{y}(s)\|_{2}\right) d s\right\} \\
& \leq \frac{K}{\epsilon} \sup _{t \leq 0}\left\{\int_{-\infty}^{t} e^{\left(\frac{-\gamma_{1}}{\epsilon}+\frac{\mu}{\epsilon}\right)(t-s)} d s\right\}\|z-\bar{z}\|_{C_{-\frac{\mu}{\epsilon}}^{-}} \\
& =\frac{K}{\gamma_{1}-\mu}\|z-\bar{z}\|_{C_{-\frac{\mu}{\epsilon}}^{-}}
\end{aligned}
$$

and

$$
\begin{aligned}
\left\|\mathcal{J}_{2}^{\epsilon}(z)-\mathcal{J}_{2}^{\epsilon}(\bar{z})\right\|_{C_{-\frac{\mu}{\epsilon}}^{2,-}} & \leq K \sup _{t \leq 0}\left\{e^{\frac{\mu}{\epsilon} t} \int_{t}^{0} e^{-\gamma_{2}(t-s)} e^{-\frac{\mu}{\epsilon} s} d s\right\}\|z-\bar{z}\|_{C_{-\frac{\mu}{\epsilon}}^{-}} \\
& \leq K \sup _{t \leq 0}\left\{\int_{t}^{0} e^{\left(-\gamma_{2}+\frac{\mu}{\epsilon}\right)(t-s)} d s\right\}\|z-\bar{z}\|_{C_{-\frac{\mu}{\epsilon}}^{-}} \\
& =\frac{\epsilon K}{\mu-\epsilon \gamma_{2}}\|z-\bar{z}\|_{C_{-\frac{\mu}{\epsilon}}^{-}} .
\end{aligned}
$$

Hence, by (4.4) and (4.5),

$$
\left\|\mathcal{J}^{\epsilon}(z)-\mathcal{J}^{\epsilon}(\bar{z})\right\|_{C_{-\frac{\mu}{\epsilon}}^{-}} \leq \kappa\left(K, \gamma_{1}, \gamma_{2}, \mu, \epsilon\right)\|z-\bar{z}\|_{C_{-\frac{\mu}{\epsilon}}^{-}}
$$

where

$$
\kappa\left(K, \gamma_{1}, \gamma_{2}, \mu, \epsilon\right)=\frac{K}{\gamma_{1}-\mu}+\frac{\epsilon K}{\mu-\epsilon \gamma_{2}} \rightarrow \frac{K}{\gamma_{1}-\mu}
$$

as $\epsilon \rightarrow 0$. Taking (3.6) into account, there is a sufficiently small constant $\epsilon_{0}>0$ such that

$$
\kappa\left(K, \gamma_{1}, \gamma_{2}, \mu, \epsilon\right)<1 \text {, for } \epsilon \in\left(0, \epsilon_{0}\right] .
$$

Therefore, the mapping $\mathcal{J}^{\epsilon}$ is strictly contractive in $C_{-\frac{\mu}{\epsilon}}^{-}$, and, consequently, the integral equation (4.1) has a unique solution $Z^{\epsilon}\left(t, \omega, Y_{0}\right)=\left(X^{\epsilon}\left(t, \omega, Y_{0}\right), Y^{\epsilon}\left(t, \omega, Y_{0}\right)\right)$ in $C_{-\frac{\mu}{\epsilon}}^{-}$. Furthermore one has the estimate

$$
\left\|Z^{\epsilon}\left(\cdot, \omega, Y_{1}\right)-Z^{\epsilon}\left(\cdot, \omega, Y_{2}\right)\right\|_{C_{-\frac{\mu}{\epsilon}}^{-}} \leq \frac{1}{1-\kappa\left(K, \gamma_{1}, \gamma_{2}, \mu, \epsilon\right)}\left\|Y_{1}-Y_{2}\right\|_{2}
$$

for all $\omega \in \Omega, Y_{1}, Y_{2} \in H_{2}$.

Defining

$$
H^{\epsilon}\left(\omega, Y_{0}\right)=\frac{1}{\epsilon} \int_{-\infty}^{0} e^{-A s / \epsilon} F\left(X^{\epsilon}\left(s, \omega, Y_{0}\right), Y^{\epsilon}\left(s, \omega, Y_{0}\right), \theta_{s}^{\epsilon} \omega\right) d s
$$


we then get from (4.6)

$$
\left\|H^{\epsilon}\left(\omega, Y_{1}\right)-H^{\epsilon}\left(\omega, Y_{2}\right)\right\|_{1} \leq \frac{K}{\left(\gamma_{1}-\mu\right)} \frac{1}{\left[1-\kappa\left(K, \gamma_{1}, \gamma_{2}, \mu, \epsilon\right)\right]}\left\|Y_{1}-Y_{2}\right\|_{2}
$$

for all $Y_{1}, Y_{2} \in H_{2}, \omega \in \Omega$. It then follows from Lemma 4.1 that

$$
M^{\epsilon}(\omega)=\left\{\left(H^{\epsilon}\left(\omega, Y_{0}\right), Y_{0}\right): Y_{0} \in H_{2}\right\} .
$$

In order to see that $M^{\epsilon}(\omega)$ is a random set we need to show that for any $z=(x, y) \in$ $\mathbb{H}=H_{1} \times H_{2}$,

$$
\omega \rightarrow \inf _{z^{\prime} \in \mathbb{H}}\left\|(x, y)-\left(H^{\epsilon}\left(\omega, \mathcal{P} z^{\prime}\right), \mathcal{P} z^{\prime}\right)\right\|
$$

is measurable; see Castaing and Valadier [7], Theorem III.9. Let $\mathbb{H}_{c}$ be a countable dense set of the separable space $\mathbb{H}$. Then the right hand side of (4.8) is equal to

$$
\inf _{z^{\prime} \in \mathbb{H}_{c}}\left\|(x, y)-\left(H^{\epsilon}\left(\omega, \mathcal{P} z^{\prime}\right), \mathcal{P} z^{\prime}\right)\right\|,
$$

which follows immediately by the continuity of $H^{\epsilon}(\omega, \cdot)$. The measurability of any expression under the infimum of (4.8) follows since $\omega \rightarrow H^{\epsilon}\left(\omega, \mathcal{P} z^{\prime}\right)$ is measurable for any $z^{\prime} \in \mathbb{H}$.

It remains to show that $M^{\epsilon}(\omega)$ is invariant, i.e., for each $Z_{0}=\left(X_{0}, Y_{0}\right) \in M^{\epsilon}(\omega)$, $Z^{\epsilon}\left(s, \omega, Z_{0}\right) \in M^{\epsilon}\left(\theta_{s}^{\epsilon} \omega\right)$ for all $s \geq 0$. We first note that for each fixed $s \geq 0, Z^{\epsilon}(t+$ $\left.s, \omega, Z_{0}\right)$ is a solution of

$$
\begin{aligned}
& d X^{\epsilon}=\frac{1}{\epsilon} A X^{\epsilon} d t+\frac{1}{\epsilon} F\left(X^{\epsilon}, Y^{\epsilon}, \theta_{t}^{\epsilon}\left(\theta_{s}^{\epsilon} \omega\right)\right) d t, \\
& d Y^{\epsilon}=B Y^{\epsilon} d t+G\left(X^{\epsilon}, Y^{\epsilon}, \theta_{t}^{\epsilon}\left(\theta_{s}^{\epsilon} \omega\right)\right) d t,
\end{aligned}
$$

with initial datum $Z(0)=(X(0), Y(0))=Z^{\epsilon}\left(s, \omega, Z_{0}\right)$. Thus, $Z^{\epsilon}\left(t+s, \omega, Z_{0}\right)=$ $Z^{\epsilon}\left(t, \theta_{s}^{\epsilon} \omega, Z^{\epsilon}\left(s, \omega, Z_{0}\right)\right)$. Since $Z^{\epsilon}\left(\cdot, \omega, Z_{0}\right) \in C_{\frac{-\mu}{\epsilon}}^{-}, Z^{\epsilon}\left(t, \theta_{s}^{\epsilon} \omega, Z^{\epsilon}\left(s, \omega, Z_{0}\right)\right) \in C_{\frac{-\mu}{\epsilon}}^{-}$. Therefore $Z^{\epsilon}\left(s, \omega, Z_{0}\right) \in M^{\epsilon}\left(\theta_{s}^{\epsilon} \omega\right)$. This completes the proof.

REMARK 4.1. We remark that the key point in the proof of Theorem 4.2 is that

$$
\kappa\left(K, \gamma_{1}, \gamma_{2}, \mu, \epsilon\right)=\frac{K}{\gamma_{1}-\mu}+\frac{\epsilon K}{\mu-\epsilon \gamma_{2}}<1 .
$$

In the particular case where $\epsilon=1$, one has $\kappa=\frac{K}{\gamma_{1}-\mu}+\frac{K}{\mu-\gamma_{2}}<1$, which is the usual spectral gap condition. We note also that the proof is valid for sufficiently small $\epsilon>0$ only in the case $\frac{K}{\gamma_{1}-\mu}<1$. This explains the assumption (A3). It is unclear to us about how to relax this condition.

In what follows we prove the exponential tracking property, which means that the manifold $M^{\epsilon}(\omega)$ attracts exponentially all the orbits of $\Phi^{\epsilon}$ if the scaling parameter is sufficiently small.

THEOREM 4.3 (Exponential tracking property). Assume that the assumptions (A1)-(A3) hold. Then for sufficiently small $\epsilon>0$, the Lipschitz invariant manifold for (3.4)-(3.5) obtained in Theorem 4.2 has the exponential tracking property 
in the following sense: There exist constants $C_{1}>0$ and $C_{2}>0$ such that for any $Z_{0}=\left(X_{0}, Y_{0}\right) \in H$ there is a $\bar{Z}_{0}=\left(\bar{X}_{0}, \bar{Y}_{0}\right) \in M^{\epsilon}(\omega)$ such that

$$
\left\|\Phi^{\epsilon}\left(t, \omega, Z_{0}\right)-\Phi^{\epsilon}\left(t, \omega, \bar{Z}_{0}\right)\right\| \leq C_{1} e^{-C_{2} t}\left\|Z_{0}-\bar{Z}_{0}\right\|, t \geq 0,
$$

where $\|\cdot\|$ denotes the norm in space $\mathbb{H}=H_{1} \times H_{2}$ defined by

$$
\|z\|=\|x\|_{1}+\|y\|_{2}, z=(x, y) .
$$

Proof. Assume that $Z^{\epsilon}(t)=\left(X^{\epsilon}(t), Y^{\epsilon}(t)\right)$ and $\bar{Z}^{\epsilon}(t)=\left(\bar{X}^{\epsilon}(t), \bar{Y}^{\epsilon}(t)\right)$ are two solutions for (3.4)-(3.5). Then $\mathcal{Z}^{\epsilon}(t)=\bar{Z}^{\epsilon}(t)-Z^{\epsilon}(t):=\left(U^{\epsilon}(t), V^{\epsilon}(t)\right)$ satisfies the equations

$$
\begin{aligned}
& d U^{\epsilon}=\frac{1}{\epsilon} A U^{\epsilon} d t+\frac{1}{\epsilon} \tilde{F}\left(U^{\epsilon}, V^{\epsilon}, \theta_{t}^{\epsilon} \omega\right) d t \\
& d V^{\epsilon}=B V^{\epsilon} d t+\tilde{G}\left(U^{\epsilon}, V^{\epsilon}, \theta_{t}^{\epsilon} \omega\right) d t
\end{aligned}
$$

where

$$
\tilde{F}\left(U^{\epsilon}, V^{\epsilon}, \theta_{t}^{\epsilon}\right)=F\left(U^{\epsilon}+X^{\epsilon}, V^{\epsilon}+Y^{\epsilon}, \theta_{t}^{\epsilon} \omega\right)-F\left(X^{\epsilon}, Y^{\epsilon}, \theta_{t}^{\epsilon} \omega\right)
$$

and

$$
\tilde{G}\left(U^{\epsilon}, V^{\epsilon}, \theta_{t}^{\epsilon}\right)=G\left(U^{\epsilon}+X^{\epsilon}, V^{\epsilon}+Y^{\epsilon}, \theta_{t}^{\epsilon} \omega\right)-G\left(X^{\epsilon}, Y^{\epsilon}, \theta_{t}^{\epsilon} \omega\right) .
$$

First we claim that $\mathcal{Z}^{\epsilon}(t)=\left(U^{\epsilon}(t), V^{\epsilon}(t)\right)$ is a solution of $(4.10)-(4.11)$ in $C_{-\frac{\mu}{\epsilon}}^{+}$if

$$
\left(\begin{array}{c}
U^{\epsilon}(t) \\
V^{\epsilon}(t)
\end{array}\right)=\left(\begin{array}{c}
e^{A t / \epsilon} U^{\epsilon}(0)+\frac{1}{\epsilon} \int_{0}^{t} e^{A(t-s)} \tilde{F}\left(U^{\epsilon}(s), V^{\epsilon}(s), \theta_{s}^{\epsilon} \omega\right) d s \\
\int_{\infty}^{t} e^{B(t-s)} \tilde{G}\left(U^{\epsilon}(s), V^{\epsilon}(s), \theta_{s}^{\epsilon} \omega\right) d s
\end{array}\right) .
$$

This can be verified by using the variation of constants formula. Next we are going to prove that (4.12) has solutions $\left(U^{\epsilon}, V^{\epsilon}\right)$ in $C_{-\frac{\mu}{\epsilon}}^{+}$with $\left(U^{\epsilon}(0), V^{\epsilon}(0)\right)=(U(0), V(0))$ and such that $\left(\bar{X}_{0}, \bar{Y}_{0}\right)=(U(0), V(0))+\left(X_{0}, Y_{0}\right) \in M^{\epsilon}(\omega)$. Recall that

$$
\left(\bar{X}_{0}, \bar{Y}_{0}\right) \in M^{\epsilon}(\omega) \Longleftrightarrow \bar{X}_{0}=\frac{1}{\epsilon} \int_{-\infty}^{0} e^{A(-s)} F\left(X^{\epsilon}\left(s, \bar{Y}_{0}\right), Y^{\epsilon}\left(s, \bar{Y}_{0}\right), \theta_{s}^{\epsilon} \omega\right) d s .
$$

It follows that

$$
\left(\bar{X}_{0}, \bar{Y}_{0}\right)=(U(0), V(0))+\left(X_{0}, Y_{0}\right) \in M^{\epsilon}(\omega)
$$

if and only if

$$
\begin{aligned}
U(0) & =-X_{0}+\frac{1}{\epsilon} \int_{-\infty}^{0} e^{A(-s)} F\left(X^{\epsilon}\left(s, V(0)+Y_{0}\right), Y^{\epsilon}\left(s, V(0)+Y_{0}\right), \theta_{s}^{\epsilon} \omega\right) d s \\
& =-X_{0}+H^{\epsilon}\left(\omega, V(0)+Y_{0}\right) .
\end{aligned}
$$

For every $\mathcal{Z}=(U, V) \in C_{-\frac{\mu}{\epsilon}}^{+}$define, for $t \geq 0$,

$$
\mathcal{I}_{1}^{\epsilon}(\mathcal{Z}(\cdot))[t]:=e^{A t / \epsilon} U(0)+\frac{1}{\epsilon} \int_{0}^{t} e^{A(t-s) / \epsilon} \tilde{F}\left(U(s), V(s), \theta_{s}^{\epsilon} \omega\right) d s
$$


and

$$
\mathcal{I}_{2}^{\epsilon}(\mathcal{Z}(\cdot))[t]:=\int_{\infty}^{t} e^{B(t-s) / \epsilon} \tilde{G}\left(U(s), V(s), \theta_{s}^{\epsilon} \omega\right) d s
$$

where $U(0)$ is given by (4.13). Define the operator $\mathcal{I}^{\epsilon}$ by

$$
\mathcal{I}^{\epsilon}(\mathcal{Z}(\cdot)):=\left(\begin{array}{c}
\mathcal{I}_{1}^{\epsilon}(\mathcal{Z}(\cdot)) \\
\mathcal{I}_{2}^{\epsilon}(\mathcal{Z}(\cdot))
\end{array}\right)
$$

It is easy to see that $\mathcal{I}^{\epsilon}$ is well-defined from $C_{-\frac{\mu}{\epsilon}}^{+}$to itself. To this end, assuming that $\mathcal{Z}, \overline{\mathcal{Z}} \in C_{-\frac{\mu}{\epsilon}}^{+}$, we obtain from (4.13) the estimate

$$
\begin{aligned}
\left\|e^{A t / \epsilon}(U(0)-\bar{U}(0))\right\|_{1} & \leq e^{-\gamma_{1} t / \epsilon} \operatorname{LipH}^{\epsilon}\|V(0)-\bar{V}(0)\|_{2} \\
& \leq e^{-\gamma_{1} t / \epsilon} \operatorname{LipH}^{\epsilon}\left\|\int_{\infty}^{0} e^{B(-s)}\left(\tilde{G}\left(\mathcal{Z}(s), \theta_{s}^{\epsilon} \omega\right)-\tilde{G}\left(\overline{\mathcal{Z}}(s), \theta_{s}^{\epsilon} \omega\right)\right) d s\right\|_{2} \\
& \leq e^{-\gamma_{1} t / \epsilon} \operatorname{LipH}^{\epsilon} \cdot K \int_{0}^{\infty} e^{\gamma_{2} s}\|\mathcal{Z}(s)-\overline{\mathcal{Z}}(s)\| d s,
\end{aligned}
$$

and so

$$
\begin{gathered}
\left\|\mathcal{I}_{1}^{\epsilon}(\mathcal{Z}-\overline{\mathcal{Z}})\right\|_{C_{-\frac{\mu}{\epsilon}}^{+, 1}} \leq L i p H^{\epsilon} \cdot K\|\mathcal{Z}-\overline{\mathcal{Z}}\|_{C_{-\frac{\mu}{\epsilon}}^{+}} \sup _{t \geq 0}\left\{e^{-\left(-\frac{\mu}{\epsilon}+\frac{\gamma_{1}}{\epsilon}\right) t} \int_{0}^{\infty} e^{\left(\gamma_{2}-\frac{\mu}{\epsilon}\right) s} d s\right\} \\
+\frac{K}{\epsilon}\|\mathcal{Z}-\overline{\mathcal{Z}}\|_{C_{-\frac{\mu}{\epsilon}}^{+}} \sup _{t \geq 0}\left\{e^{\frac{\mu}{\epsilon} t} \int_{0}^{t} e^{-\gamma_{1}(t-s) / \epsilon} e^{-\frac{\mu}{\epsilon} s} d s\right\} \\
\leq\left(\frac{L i p H^{\epsilon} \cdot \epsilon K}{\mu-\epsilon \gamma_{2}}+\frac{K}{\gamma_{1}-\mu}\right)\|\mathcal{Z}-\overline{\mathcal{Z}}\|_{C_{-\frac{\mu}{\epsilon}}^{+}}
\end{gathered}
$$

For the operator $\mathcal{I}_{2}^{\epsilon}$ we have

$$
\begin{aligned}
\left\|\mathcal{I}_{2}^{\epsilon}(\mathcal{Z}-\overline{\mathcal{Z}})\right\|_{C_{-\frac{\mu}{\epsilon}}^{+, 2}} & \leq K\|\mathcal{Z}-\overline{\mathcal{Z}}\|_{C_{-\frac{\mu}{\epsilon}}^{+}} \sup _{t \geq 0}\left\{e^{-\left(-\frac{\mu}{\epsilon}+\gamma_{2}\right) t} \int_{t}^{\infty} e^{\left(-\frac{\mu}{\epsilon}+\gamma_{2}\right) s} d s\right\} \\
& \leq \frac{\epsilon K}{\mu-\gamma_{2}}\|\mathcal{Z}-\overline{\mathcal{Z}}\|_{C_{-\frac{\mu}{\epsilon}}^{+}}
\end{aligned}
$$

Recalling that

$$
L i p H^{\epsilon} \leq \frac{K}{\left(\gamma_{1}-\mu\right)\left[1-K\left(\frac{1}{\gamma_{1}-\mu}+\frac{\epsilon}{\mu-\epsilon \gamma_{2}}\right)\right]},
$$

and taking (4.14) and (4.15) into account, we obtain

$$
\left\|\mathcal{I}^{\epsilon}(\mathcal{Z}-\overline{\mathcal{Z}})\right\|_{C_{-\frac{\mu}{\epsilon}}^{+}} \leq \rho\left(K, \gamma_{1}, \gamma_{2}, \mu, \epsilon\right)\|\mathcal{Z}-\overline{\mathcal{Z}}\|_{C_{-\frac{\mu}{\epsilon}}^{+}}
$$

with

$$
\begin{aligned}
\rho\left(K, \gamma_{1}, \gamma_{2}, \mu, \epsilon\right)=\frac{K}{\gamma_{1}-\mu} & +\frac{\epsilon K}{\mu-\epsilon \gamma_{2}} \\
& +\frac{K^{2}}{\left(\gamma_{1}-\mu\right)\left(\frac{\mu}{\epsilon}-\gamma_{2}\right)\left[1-K\left(\frac{1}{\gamma_{1}-\mu}+\frac{\epsilon}{\mu-\epsilon \gamma_{2}}\right)\right]}
\end{aligned}
$$




$$
\rightarrow \frac{K}{\gamma_{1}-\mu}
$$

as $\epsilon \rightarrow 0$. By (3.6) there is a sufficiently small constant $\epsilon_{0}^{\prime}>0$ such that $\rho\left(K, \gamma_{1}, \gamma_{2}, \mu, \epsilon\right)<1$ for all $0<\epsilon<\epsilon_{0}^{\prime}$. Therefore, the operator $\mathcal{I}^{\epsilon}$ is strictly contractive and has a unique fixed point $\mathcal{Z} \in C_{-\frac{\mu}{\epsilon}}^{+}$, which is the unique solution for (4.12) and satisfies $\left(\bar{X}_{0}, \bar{Y}_{0}\right)=(U(0), V(0))+\left(X_{0}, Y_{0}\right) \in M^{\epsilon}(\omega)$. Moreover, we have

$$
\|\mathcal{Z}\|_{C_{-\frac{\mu}{\epsilon}}^{+}} \leq \frac{1}{1-\left(\frac{K}{\gamma_{1}-\mu}+\frac{\epsilon K}{\mu-\epsilon \gamma_{2}}\right)}\|\mathcal{Z}(0)\|
$$

which means

$$
\left\|\Phi^{\epsilon}\left(t, \omega, Z_{0}\right)-\Phi^{\epsilon}\left(t, \omega, \bar{Z}_{0}\right)\right\| \leq \frac{e^{-\frac{\mu}{\epsilon} t}}{1-\left(\frac{K}{\gamma_{1}-\mu}+\frac{\epsilon K}{\mu-\epsilon \gamma_{2}}\right)}\left\|Z_{0}-\bar{Z}_{0}\right\|, t>0 .
$$

Therefore, the exponential tracking property of $M^{\epsilon}(\omega)$ is obtained.

REMARK 4.2. By the relationship between the solutions of system (3.1)-(3.2) and (3.4)-(3.5), the original fast-slow stochastic system also has a Lipschitz random invariant manifold under the conditions of Theorem 4.2, which is represented as

$$
\begin{aligned}
\mathcal{M}^{\epsilon}(\omega) & =M^{\epsilon}(\omega)+\left(\eta^{\frac{1}{\epsilon}}(\omega), 0\right) \\
& =\left\{\left(h^{\epsilon}\left(\omega, Y_{0}\right), Y_{0}\right): Y_{0} \in H_{2}\right\},
\end{aligned}
$$

with

$$
h^{\epsilon}\left(\omega, Y_{0}\right)=H^{\epsilon}\left(\omega, Y_{0}\right)+\eta^{\frac{1}{\epsilon}}(\omega) .
$$

Hence, if system (3.4)-(3.5) has an exponential tracking manifold, then so has system $(3.1)-(3.2)$.

REMARK 4.3. Theorem 4.3 implies that any orbit of the fast-slow system tends exponentially to an orbit on the manifold $M^{\epsilon}(\omega)$ which is governed by an evolutionary equation with usual time scale. To be more specific, for any solution $Z^{\epsilon}=\left(X^{\epsilon}, Y^{\epsilon}\right)$ for (3.4)-(3.5), there is an orbit $\tilde{Z}^{\epsilon}(t, \omega)=\left(\tilde{X}^{\epsilon}(t, \omega), \tilde{Y}^{\epsilon}(t, \omega)\right)$ on the manifold $M^{\epsilon}$ which satisfies the evolutionary equation

$$
\dot{\tilde{Y}}^{\epsilon}=B \tilde{Y}^{\epsilon}+G\left(H^{\epsilon}\left(\theta_{t}^{\epsilon} \omega, \tilde{Y}^{\epsilon}\right), \tilde{Y}^{\epsilon}, \theta_{t}^{\epsilon} \omega\right)
$$

such that

$$
\left\|Z^{\epsilon}(t, \omega)-\tilde{Z}^{\epsilon}(t, \omega)\right\| \leq \frac{e^{-\frac{\mu}{\epsilon} t}}{1-\left(\frac{K}{\gamma_{1}-\mu}+\frac{\epsilon K}{\mu-\epsilon \gamma_{2}}\right)}\left\|Z_{0}-\tilde{Z}_{0}\right\|, t>0,
$$

where $Z_{0}=\left(X^{\epsilon}(0), Y^{\epsilon}(0)\right)$ and $\tilde{Z}_{0}=(\tilde{X}(0), \tilde{Y}(0))$. Applying the ideas from the Remark 4.2 , we have a reduction system which describes the long-time behavior for system (3.1)-(3.2). 
TheOrem 4.4 (Reduction system). Assume that $\epsilon>0$ is sufficiently small and that the assumptions $(\boldsymbol{A} \mathbf{1})-(\boldsymbol{A} 3)$ hold. Then for any solution $z^{\epsilon}(t)=\left(x^{\epsilon}(t), y^{\epsilon}(t)\right)$ to system (3.1)-(3.2) with initial data $z^{\epsilon}(0)=\left(x_{0}, y_{0}\right)$, there exists a solution $\tilde{z}^{\epsilon}(t)=$ $\left(\tilde{x}^{\epsilon}(t), \tilde{y}^{\epsilon}(t)\right)$ with $\tilde{z}(0)=\left(\tilde{x}^{\epsilon}(0), \tilde{y}^{\epsilon}(0)\right)=\left(\tilde{x}_{0}, \tilde{y}_{0}\right)$ to the reduced system

$$
\left\{\begin{array}{l}
\dot{\tilde{y}}^{\epsilon}=B \tilde{y}^{\epsilon}+g\left(\tilde{x}, \tilde{y}^{\epsilon}\right), \\
\tilde{x}=h^{\epsilon}\left(\theta_{t}^{\epsilon} \omega, \tilde{y}^{\epsilon}\right),
\end{array}\right.
$$

such that for any $t \geq 0$ and almost sure $\omega \in \Omega$,

$$
\begin{aligned}
\left\|z^{\epsilon}(t, \omega)-\tilde{z}^{\epsilon}(t, \omega)\right\| & \leq \frac{e^{-\frac{\mu}{\epsilon} t}}{1-\left(\frac{K}{\gamma_{1}-\mu}+\frac{\epsilon K}{\mu-\epsilon \gamma_{2}}\right)}\left\|z_{0}-\tilde{z}_{0}\right\| \\
& \leq C_{K, \gamma_{1}, \mu} e^{\frac{-\mu t}{\epsilon}}\left\|z_{0}-\tilde{z}_{0}\right\|,
\end{aligned}
$$

with $\frac{-\mu}{\epsilon}<0$ and $C_{K, \gamma_{1}, \mu}$ being a constant depending on $K, \gamma_{1}$, and $\mu$.

\section{Slow manifolds}

Now we consider an asymptotic approximation for the invariant manifold $M^{\epsilon}(\omega)$, as $\epsilon \rightarrow 0$.

The scaling $t \rightarrow \epsilon t$ in system (3.4)-(3.5) yields

$$
\begin{aligned}
& d X^{\epsilon}=A X^{\epsilon} d t+F\left(X^{\epsilon}, Y^{\epsilon}, \theta_{\epsilon t}^{\epsilon} \omega\right) d t, \\
& d Y^{\epsilon}=\epsilon B Y^{\epsilon} d t+\epsilon G\left(X^{\epsilon}, Y^{\epsilon}, \theta_{\epsilon t}^{\epsilon} \omega\right) d t,
\end{aligned}
$$

where

$$
\begin{aligned}
& F\left(X^{\epsilon}, Y^{\epsilon}, \theta_{\epsilon t}^{\epsilon} \omega\right)=f\left(X^{\epsilon}+\eta^{\frac{1}{\epsilon}}\left(\theta_{\epsilon t} \omega\right), Y^{\epsilon}\right), \\
& G\left(X^{\epsilon}, Y^{\epsilon}, \theta_{\epsilon t}^{\epsilon} \omega\right)=g\left(X^{\epsilon}+\eta^{\frac{1}{\epsilon}}\left(\theta_{\epsilon t} \omega\right), Y^{\epsilon}\right) .
\end{aligned}
$$

We now replace $\eta^{\frac{1}{\epsilon}}\left(\theta_{\epsilon t} \omega\right)$ by $\xi\left(\theta_{t} \omega\right)$, which has the same distribution, so that we have a random evolutionary system whose solution's distribution coincides with that of the system (5.1)-(5.2), in the form of

$$
\begin{aligned}
& d \breve{X}^{\epsilon}=A \breve{X}^{\epsilon} d t+f\left(\breve{X}^{\epsilon}+\xi\left(\theta_{t} \omega\right), \breve{Y}^{\epsilon}\right) d t, \\
& d \breve{Y}^{\epsilon}=\epsilon B \breve{Y}^{\epsilon} d t+\epsilon g\left(\breve{X}^{\epsilon}+\xi\left(\theta_{t} \omega\right), \breve{Y}^{\epsilon}\right) d t .
\end{aligned}
$$

By proceeding as in the proof of Theorem 4.2, it can be shown that the system (5.3)(5.4) has a random invariant manifold represented as

$$
\breve{M}^{\epsilon}(\omega)=\left\{\left(\breve{H}^{\epsilon}\left(\omega, Y_{0}\right), Y_{0}\right): Y_{0} \in H_{2}\right\}
$$

with

$$
\breve{H}^{\epsilon}\left(\omega, Y_{0}\right)=\int_{-\infty}^{0} e^{A s} f\left(\breve{X}^{\epsilon}\left(s, \omega, Y_{0}\right)+\xi\left(\theta_{t} \omega\right), \breve{Y}^{\epsilon}\left(s, \omega, Y_{0}\right)\right) d s
$$

where

$$
\begin{aligned}
& \breve{X}^{\epsilon}\left(t, \omega, Y_{0}\right)=\int_{-\infty}^{t} e^{A(t-s)} f\left(\breve{X}^{\epsilon}\left(s, \omega, Y_{0}\right)+\xi\left(\theta_{s} \omega\right), \breve{Y}^{\epsilon}\left(s, \omega, Y_{0}\right)\right) d s, t \leq 0, \\
& \breve{Y}^{\epsilon}\left(t, \omega, Y_{0}\right)=e^{B t \epsilon} Y_{0}+\epsilon \int_{0}^{t} e^{B(t-s) \epsilon} g\left(\breve{X}^{\epsilon}\left(s, \omega, Y_{0}\right)+\xi\left(\theta_{s} \omega\right), \breve{Y}^{\epsilon}\left(s, \omega, Y_{0}\right)\right) d s, t \leq 0
\end{aligned}
$$


is the unique solution in $C_{-\mu}^{-}$for the above integral equations. With a change of variables $s / \epsilon \rightarrow t$ in (4.7), we have

$$
\begin{aligned}
H^{\epsilon}\left(\omega, Y_{0}\right) & =\int_{-\infty}^{0} e^{-A s} f\left(X^{\epsilon}\left(s \epsilon, \omega, Y_{0}\right)+\eta^{\frac{1}{\epsilon}}\left(\theta_{\epsilon \epsilon} \omega\right), Y^{\epsilon}\left(s \epsilon, \omega, Y_{0}\right)\right) d s \\
& =\int_{-\infty}^{0} e^{-A s} f\left(X^{\epsilon}\left(s \epsilon, \omega, Y_{0}\right)+\eta^{\frac{1}{\epsilon}}\left(\theta_{\epsilon \epsilon} \omega\right), Y^{\epsilon}\left(s \epsilon, \omega, Y_{0}\right)\right) d s \\
& \simeq \breve{H}^{\epsilon}\left(\omega, Y_{0}\right),
\end{aligned}
$$

where $\simeq$ denotes equivalence (coincidence) in distribution. Therefore, the invariant manifold $\breve{M}^{\epsilon}(\omega)$ is a version in distribution for $M^{\epsilon}(\omega)$.

Next, we show that there exists a random invariant manifold $M^{0}(\omega)$, which is called a random slow manifold for system (5.3)-(5.4), and which will be the asymptotic limit of the manifold $\breve{M}^{\epsilon}(\omega)$ as $\epsilon \rightarrow 0$. To this end, we consider the system

$$
\begin{aligned}
d \bar{X} & =A \bar{X} d t+f\left(\bar{X}+\xi\left(\theta_{t} \omega\right), \bar{Y}\right) d t \\
d \bar{Y} & =0
\end{aligned}
$$

By the same discussion as in Theorem 4.2, system (5.5)-(5.6) has a random invariant manifold with representation

$$
\bar{M}^{0}(\omega)=\left\{\left(\bar{H}^{0}\left(\omega, Y_{0}\right), Y_{0}\right): Y_{0} \in H_{2}\right\},
$$

where

$$
\bar{H}^{0}\left(\omega, Y_{0}\right)=\int_{-\infty}^{0} e^{-A s} f\left(\bar{X}\left(s, \omega, Y_{0}\right)+\xi\left(\theta_{s} \omega\right), Y_{0}\right) d s
$$

and $\bar{X}\left(t, \omega, Y_{0}\right)$ is the unique solution in $C_{-\mu}^{1,-}$ for the integral equation

$$
\bar{X}\left(t, \omega, Y_{0}\right)=\int_{-\infty}^{t} e^{A(t-s)} f\left(\bar{X}\left(s, \omega, Y_{0}\right)+\xi\left(\theta_{s} \omega\right), Y_{0}\right) d s, t \leq 0
$$

The main result of this section is the following theorem.

THeOREM 5.1 (Slow manifolds). Let the assumptions (A1)-(A3) hold and also assume that there exists a positive number $C_{g}$ such that $\sup _{x \in H_{1}, y \in H_{2}}\|g(x, y)\|_{H_{2}}=C_{g}$. Then the invariant manifold $\breve{M}^{\epsilon}(\omega)$ for the system (5.1)-(5.2) can be approximated by a slow manifold $\bar{M}^{0}(\omega)$ defined in (5.7), in the sense that their respective graph mappings $\breve{H}^{\epsilon}$ and $\bar{H}$ satisfy

$$
\left\|\breve{H}^{\epsilon}\left(\omega, Y_{0}\right)-\bar{H}\left(\omega, Y_{0}\right)\right\|_{1}=\mathcal{O}(\epsilon),
$$

or

$$
\breve{H}^{\epsilon}\left(\omega, Y_{0}\right)=\bar{H}\left(\omega, Y_{0}\right)+\mathcal{O}(\epsilon)
$$

for all $Y_{0} \in \mathcal{D}(B)$, a.s. $\omega \in \Omega$, and as $\epsilon \rightarrow 0$.

Proof. In this proof, the letter $C$ with or without subscripts denotes positive constants whose value may change in different occasions. We will write the dependence 
of this constant on parameters explicitly if it is essential. As is known [28], if $Y_{0} \in \mathcal{D}(B)$ and $t \leq 0$,

$$
\begin{aligned}
\left\|e^{B t \epsilon} Y_{0}-Y_{0}\right\|_{2} & =\left\|\int_{\epsilon t}^{0} e^{B \tau} B Y_{0} d \tau\right\|_{2} \\
& \leq\left\|B Y_{0}\right\|_{2} \int_{\epsilon t}^{0} e^{-\gamma_{2} \tau} d \tau \\
& =\left\|B Y_{0}\right\|_{2} \frac{1}{\gamma_{2}}\left(e^{-\gamma_{2} \epsilon t}-1\right) .
\end{aligned}
$$

Then we have, for all $t \leq 0$,

$$
\begin{aligned}
\left\|\breve{Y}^{\epsilon}\left(t, \omega, Y_{0}\right)-Y_{0}\right\|_{2} \leq & \left\|e^{B \epsilon t} Y_{0}-Y_{0}\right\|_{2} \\
& +\epsilon\left\|\int_{t}^{0} e^{B \epsilon(t-s)} g\left(\breve{X}^{\epsilon}\left(s, \omega, Y_{0}\right)+\xi\left(\theta_{t} \omega\right), \breve{Y}^{\epsilon}\left(s, \omega, Y_{0}\right)\right) d s\right\|_{2} \\
& \leq\left\|B Y_{0}\right\|_{2} \frac{1}{\gamma_{2}}\left(e^{-\gamma_{2} \epsilon t}-1\right)+\epsilon C_{g} \int_{t}^{0} e^{-\epsilon \gamma_{2}(t-s)} d s \\
& =C\left(e^{-\gamma_{2} \epsilon t}-1\right) .
\end{aligned}
$$

Then, by again using (5.8), we have

$$
\begin{gathered}
\left\|\breve{X}^{\epsilon}\left(t, \omega, Y_{0}\right)-\bar{X}\left(t, \omega, Y_{0}\right)\right\|_{1} \leq K \int_{-\infty}^{t} e^{-\gamma_{1}(t-s)}\left\|\breve{X}^{\epsilon}\left(s, \omega, Y_{0}\right)-\bar{X}\left(s, \omega, Y_{0}\right)\right\|_{1} d s \\
+K C \int_{-\infty}^{t} e^{-\gamma_{1}(t-s)}\left(e^{-\gamma_{2} \epsilon t}-1\right) d s \\
=K \int_{-\infty}^{t} e^{-\gamma_{1}(t-s)}\left\|\breve{X}^{\epsilon}\left(s, \omega, Y_{0}\right)-\bar{X}\left(s, \omega, Y_{0}\right)\right\|_{1} d s \\
+C\left(\frac{1}{\gamma_{1}-\epsilon \gamma_{2}} e^{-\epsilon \gamma_{2} t}-\frac{1}{\gamma_{1}}\right),
\end{gathered}
$$

which implies

$$
\begin{aligned}
& \left\|\breve{X}^{\epsilon}\left(\cdot, \omega, Y_{0}\right)-\bar{X}\left(\cdot, \omega, Y_{0}\right)\right\|_{C_{-\mu}^{1,-}} \\
\leq & K\left\|\breve{X}^{\epsilon}\left(\cdot, \omega, Y_{0}\right)-\bar{X}\left(\cdot, \omega, Y_{0}\right)\right\|_{C_{-\mu}^{1,-} \cdot} \sup _{t \leq 0} \int_{-\infty}^{t} e^{-\left(\gamma_{1}-\mu\right)(t-s)} d s \\
& +C \sup _{t \leq 0}\left\{e^{\mu t}\left(\frac{1}{\gamma_{1}-\epsilon \gamma_{2}} e^{-\epsilon \gamma_{2} t}-\frac{1}{\gamma_{1}}\right)\right\} \\
= & \frac{K}{\gamma_{1}-\mu}\left\|\breve{X}^{\epsilon}\left(\cdot, \omega, Y_{0}\right)-\bar{X}\left(\cdot, \omega, Y_{0}\right)\right\|_{C_{-\mu}^{1,-}}+C \sup _{t \leq 0} \mathscr{S}(t, \epsilon),
\end{aligned}
$$

where

$$
\mathscr{S}(t, \epsilon)=e^{\mu t}\left(\frac{1}{\gamma_{1}-\epsilon \gamma_{2}} e^{-\epsilon \gamma_{2} t}-\frac{1}{\gamma_{1}}\right), t \in(-\infty, 0],
$$

with

$$
\mathscr{S}(0, \epsilon)=\frac{1}{\gamma_{1}-\epsilon \gamma_{2}}-\frac{1}{\gamma_{1}} .
$$


Furthermore, for sufficiently small $\epsilon>0$,

$$
\begin{aligned}
\frac{d \mathscr{S}(t, \epsilon)}{d t} & =e^{\mu t}\left(\frac{\mu-\epsilon \gamma_{2}}{\gamma_{1}-\epsilon \gamma_{2}} e^{-\epsilon \gamma_{2} t}-\frac{\mu}{\gamma_{1}}\right) \\
& \leq e^{\mu t}\left(\frac{\mu-\epsilon \gamma_{2}}{\gamma_{1}-\epsilon \gamma_{2}}-\frac{\mu}{\gamma_{1}}\right) \\
& =e^{\mu t} \frac{-\epsilon \gamma_{2}\left(\gamma_{1}-\mu\right)}{\gamma_{1}\left(\gamma_{1}-\epsilon \gamma_{2}\right)} \\
& <0, t \in(-\infty, 0) .
\end{aligned}
$$

Now, according to (5.10), (5.11), and (5.12), we have

$$
\begin{aligned}
\left\|\breve{X}\left(\cdot, \omega, Y_{0}\right)-\bar{X}\left(\cdot, \omega, Y_{0}\right)\right\|_{C_{-\mu}^{1,-}} \leq & \frac{K}{\gamma_{1}-\mu}\left\|\breve{X}^{\epsilon}\left(\cdot, \omega, Y_{0}\right)-\bar{X}\left(\cdot, \omega, Y_{0}\right)\right\|_{C_{-\mu}^{1,-}} \\
& +C\left(\frac{1}{\gamma_{1}-\epsilon \gamma_{2}}-\frac{1}{\gamma_{1}}\right) .
\end{aligned}
$$

By (3.6),

$$
\left\|\breve{X}^{\epsilon}\left(\cdot, \omega, Y_{0}\right)-\bar{X}\left(\cdot, \omega, Y_{0}\right)\right\|_{C_{-\mu}^{1,-}} \leq C\left(\frac{1}{\gamma_{1}-\epsilon \gamma_{2}}-\frac{1}{\gamma_{1}}\right) .
$$

Hence, thanks to (5.9) and (5.13), we deduce

$$
\begin{aligned}
\left\|\breve{H}^{\epsilon}\left(\omega, Y_{0}\right)-\bar{H}\left(\omega, Y_{0}\right)\right\|_{1} \leq K \int_{-\infty}^{0} e^{\gamma_{1} s}\left\|\breve{X}^{\epsilon}\left(s, \omega, Y_{0}\right)-\bar{X}\left(s, \omega, Y_{0}\right)\right\|_{1} d s \\
\quad+K \int_{-\infty}^{0} e^{\gamma_{1} s}\left\|\breve{Y}^{\epsilon}\left(s, \omega, Y_{0}\right)-Y_{0}\right\|_{2} d s \\
\leq C\left(\frac{1}{\gamma_{1}-\epsilon \gamma_{2}}-\frac{1}{\gamma_{1}}\right) \int_{-\infty}^{0} e^{\left(\gamma_{1}-\mu\right) s} d s \\
\quad+C \int_{-\infty}^{0} e^{\gamma_{1} s}\left(e^{-\gamma_{2} \epsilon s}-1\right) d s \\
=C\left(\frac{1}{\gamma_{1}-\epsilon \gamma_{2}}-\frac{1}{\gamma_{1}}\right)=\mathcal{O}(\epsilon) .
\end{aligned}
$$

This completes the proof.

Remark 5.1. Consider the case where $H_{2}$ is a finite dimensional space, the operator $B$ is a constant matrix, and (5.3)-(5.4) is a coupled system of an evolutionary equation and ordinary differential equations. This type of system arises from biology, such as Hodgkin-Huxley systems (see Example 6.2). Then the above theorem implies that for any bounded set $E \subset H_{2}$,

$$
\sup _{Y_{0} \in E}\left\|\breve{H}^{\epsilon}\left(\omega, Y_{0}\right)-\bar{H}\left(\omega, Y_{0}\right)\right\|_{1}=\mathcal{O}(\epsilon), \text { a.s. } \omega \in \Omega \text { as } \epsilon \rightarrow 0 .
$$

\section{Illustrative examples}

Let us look at several examples to illustrate the results in the previous two sections. 
ExAmple 6.1. Let $D \subset \mathbb{R}^{3}$ be a bounded domain with smooth boundary $\partial D$. Consider a coupled system of stochastic parabolic-hyperbolic partial differential equations (see, e.g., [6] and [16])

$$
\begin{aligned}
& u_{t}=\frac{1}{\epsilon}(\Delta u-\alpha u)+\frac{1}{\epsilon} f\left(u, v, v_{t}\right)+\frac{1}{\sqrt{\epsilon}} \dot{w}(t), \\
& u=0 \text { on } \partial \mathrm{D}, \\
& v_{t t}=\Delta v-\beta v+g\left(u, v, v_{t}\right), \\
& v=0 \text { on } \partial \mathrm{D},
\end{aligned}
$$

where $\Delta$ denotes the Laplace operator and the parameters $\alpha, \beta$ are positive. The interaction functions

$$
f: \mathbb{R}^{3} \mapsto \mathbb{R} \text { and } g: \mathbb{R}^{3} \mapsto \mathbb{R}
$$

are assumed to be Lipschitz continuous with a Lipschitz constant $K>0$. Thus the assumption (A2) holds. Such a system may describe a thermoelastic wave propagation in a random medium [10]. The wave profile $v$ in an interacting random thermoelastic medium is described by a hyperbolic partial differential equation. If the wave is temperature dependent and the heat conductivity has faster evolution, then the hyperbolic equation is coupled to a stochastic parabolic (heat) equation with different characteristic timescales.

We introduce the usual Hilbert space $L^{2}(D)$ as well as the Sobolev spaces $H^{2}(D)$ and $H_{0}^{1}(D)$. Take $H_{1}=L^{2}(D)$. Let $A=\Delta-\alpha I_{i d}$ with domain $\mathcal{D}(A)=H^{2} \cap H_{0}^{1}$. By the semigroup theory the operator $A$ generates a contraction semigroup $\left\{e^{A t}: t \geq 0\right\}$ in $H_{1}$ ([28]) which satisfies $\left\|e^{A t}\right\|_{H_{1}} \leq e^{-\gamma_{1} t}, t \geq 0$ with $\gamma_{1}=\alpha$. Let $B=\Delta-\beta I_{i d}$ with domain $D(B)=H^{2} \cap H_{0}^{1}$. Define

$$
z:=\left(\begin{array}{c}
v \\
v^{\prime}
\end{array}\right), \mathcal{B}:=\left(\begin{array}{cc}
0 & I_{i d} \\
B & 0
\end{array}\right),
$$

and $H_{2}=H_{0}^{1}(D) \times L^{2}(D)$ with the energy norm

$$
\|z\|_{H_{2}}=\left\{\|v\|_{H_{0}^{1}}^{2}+\left\|v^{\prime}\right\|_{L^{2}}^{2}\right\}^{\frac{1}{2}},
$$

where $\|\cdot\|_{H_{0}^{1}}$ and $\|\cdot\|_{L^{2}}$ denote the norms in $H_{0}^{1}$ and $L^{2}$, respectively. Let $\mathcal{D}(\mathcal{B})=$ $\mathcal{D}(B) \times H^{1}$. It is known that $\mathcal{B}$ generates a unitary group ([33]) in $H_{2}$ which satisfies $\left\|e^{\mathcal{B} t}\right\|_{H_{2}} \leq e^{-\gamma_{2} t}$ for $t \in \mathbb{R}$ with $\gamma_{2}=0$. Then the system (6.1)-(6.4) can be rewritten as

$$
\begin{aligned}
& u_{t}=\frac{1}{\epsilon} A u+f(u, z)+\frac{1}{\sqrt{\epsilon}} \dot{w}_{t}, \\
& z_{t}=\mathcal{B} z+G(u, z),
\end{aligned}
$$

with

$$
G(u, z)=(0, g(u, z)),
$$

which is in the standard form of (3.1)-(3.2). Thus under the condition

$$
K<\gamma_{1},
$$


and if the scaling parameter $\epsilon$ is small enough, the random dynamical system generated by (6.1)-(6.4) has an invariant manifold $\mathcal{M}^{\epsilon}(\omega)=\left\{\left(h^{\epsilon}\left(\omega, Y_{0}\right), Y_{0}\right) Y_{0} \in H_{2}\right\}$, which possesses the exponential tracking property by Theorem 4.3. Moreover, by Theorem 4.4 , the reduction system for long-time behavior to system (6.1)-(6.4) is

$$
\left\{\begin{array}{l}
\dot{\tilde{y}}^{\epsilon}=\mathcal{B} \tilde{y}^{\epsilon}+G\left(\tilde{x}, \tilde{y}^{\epsilon}\right), \\
\tilde{x}=h^{\epsilon}\left(\theta_{t}^{\epsilon} \omega, \tilde{y}^{\epsilon}\right) .
\end{array}\right.
$$

Note that a similar result for this example has also been obtained in [6].

EXAMPLE 6.2. Let $D \subset \mathbb{R}^{n}$ be a bounded domain with smooth boundary $\partial D$. Consider a system of coupled parabolic partial differential equations and ordinary differential equations

$$
\begin{aligned}
& u_{t}=\frac{1}{\epsilon} \Delta u+\frac{1}{\epsilon} f(u, v)+\frac{1}{\sqrt{\epsilon}} \dot{w}_{t}, \\
& u=0 \text { on } \partial \mathrm{D}, \\
& v_{t}=g(u, v),
\end{aligned}
$$

where $f: \mathbb{R}^{1+m} \mapsto \mathbb{R}, g: \mathbb{R}^{1+m} \mapsto \mathbb{R}^{m}$ are Lipschitz maps with a Lipschitz constant $K>$ 0 :

$$
\begin{aligned}
& \left|f\left(x_{1}, y_{1}\right)-f\left(x_{2}, y_{2}\right)\right| \leq K\left(\left|x_{1}-x_{2}\right|+\left|y_{1}-y_{2}\right|_{\mathbb{R}^{m}}\right), \\
& \left|g\left(x_{1}, y_{1}\right)-g\left(x_{2}, y_{2}\right)\right|_{\mathbb{R}^{m}} \leq K\left(\left|x_{1}-x_{2}\right|+\left|y_{1}-y_{2}\right|_{\mathbb{R}^{m}}\right),
\end{aligned}
$$

for all $(x, y) \in \mathbb{R} \times \mathbb{R}^{m}$. Thus the assumption (A2) holds. This system may model certain biological processes. For instance, the famous FitzHugh-Nagumo system [19, 27], as a simplified version of the Hodgkin-Huxley model [13], which describes mechanisms of a neural excitability and excitation for macro-receptors, belongs to this class.

As in Example 6.1 the differential operator $A=\Delta$ with domain $\mathcal{D}(A)=H^{2} \cap H_{0}^{1}$ generates a $C_{0}$-semigroup $\left\{e^{A t}: t \geq 0\right\}$ on $H_{1}=L^{2}(D)$ which satisfies $\left\|e^{A t}\right\|_{H_{1}} \leq e^{-\gamma_{1} t}$ with $\gamma_{1}=\inf \operatorname{spec}\{A\}>0$. Let $B \equiv 0$ in $H_{2}=\left[L^{2}(D)\right]^{m}$. It is clear that $e^{B t}=I_{i d}$ for all $t \in \mathbb{R}$ and $\left\|e^{B t}\right\|_{H_{2}} \leq e^{-\gamma_{2} t}$ with $\gamma_{2}=0$. Therefore, the system (6.5)-(6.7) has a random invariant manifold $\mathcal{M}^{\epsilon}(\omega)=\left\{\left(h^{\epsilon}\left(\omega, Y_{0}\right), Y_{0}\right) Y_{0} \in H_{2}\right\}$ with an exponential tracking property if $K<\gamma_{1}$ and $\epsilon>0$ is small enough. We also have the reduction system

$$
\left\{\begin{array}{l}
\dot{\tilde{y}}^{\epsilon}=g\left(\tilde{x}, \tilde{y}^{\epsilon}\right), \\
\tilde{x}=h^{\epsilon}\left(\theta_{t}^{\epsilon} \omega, \tilde{y}^{\epsilon}\right),
\end{array}\right.
$$

for the long time behavior of the original system (6.5)-(6.7).

Example 6.3. Consider the following system of two coupled wave equations (i.e., hyperbolic partial differential equations) on a bounded spatial interval $I=[0, \pi]$ :

$$
\begin{aligned}
& u_{t t}=\frac{1}{\epsilon}\left(\Delta u-\nu u_{t}\right)+\frac{1}{\epsilon} f\left(u, v, v_{t}\right)+\frac{1}{\sqrt{\epsilon}} \dot{w}(t), \\
& u=0 \text { on } \partial \mathrm{I} \\
& v_{t t}=\Delta v-\beta v+g\left(u, v, v_{t}\right) \\
& v=0 \text { on } \partial \mathrm{I}
\end{aligned}
$$


where $\Delta$ denotes the Laplace operator and the parameters $\beta, \nu$ are positive. The interaction functions

$$
f: \mathbb{R}^{3} \mapsto \mathbb{R} \text { and } g: \mathbb{R}^{3} \mapsto \mathbb{R}
$$

are Lipschitz continuous with a Lipschitz constant $K>0$. Thus the assumption (A2) holds. This system models, for example, vibrating strings connected in parallel with zero boundary conditions [26] and multi-component wave fields such as electromagnetic waves in plasmas, elastic waves in solids, and light waves in anisotropic and inhomogeneous media [22].

Rewrite the equations (6.8)-(6.9) as

$$
\frac{d U}{d t}=\frac{1}{\epsilon} \mathcal{A}^{\epsilon} U+\frac{1}{\epsilon} F(U, V)+\frac{1}{\sqrt{\epsilon}} \dot{W}(t)
$$

where

$$
\mathcal{A}^{\epsilon}=\left(\begin{array}{cc}
0 & \epsilon I_{i d} \\
\Delta & -\nu
\end{array}\right), F(U, V)=\left(\begin{array}{c}
0 \\
f\left(u, v, v^{\prime}\right)
\end{array}\right), \dot{W}(t)=\left(\begin{array}{c}
0 \\
\dot{w}(t)
\end{array}\right)
$$

and

$$
U=\left(u, u^{\prime}\right), V=\left(v, v^{\prime}\right) \in H_{0}^{1}(0, \pi) \times L^{2}(0, \pi) .
$$

The linear operator $\mathcal{A}^{\epsilon}$ has the eigenvalues

$$
\lambda_{k}^{ \pm}=\frac{\nu \pm \sqrt{\nu^{2}-4 k^{2} \epsilon}}{2}, k=1,2, \ldots
$$

with the corresponding eigenvectors

$$
e_{k}^{ \pm}=\left(\begin{array}{c}
\sin k x \\
\lambda_{k}^{ \pm} \sin k x
\end{array}\right)
$$

It is clear that the operator $\mathcal{A}^{\epsilon}$ generates a $C_{0}$-semigroup $e^{\mathcal{A}^{\epsilon} t}$ on the Hilbert space $H_{1}:=H_{0}^{1}(0, \pi) \times L^{2}(0, \pi)$ equipped with energy norm introduced in Example 6.1, and it satisfies

$$
\left\|e^{\mathcal{A}^{\epsilon} t}\right\|_{H_{1}} \leq e^{-\nu t}, t \geq 0 .
$$

In the same way as in Example 6.1 the linear part of the equation (6.10)-(6.11) generates a unitary $C_{0}$-semigroup on the Hilbert space $H_{2}=H_{0}^{1}(0, \pi) \times L^{2}(0, \pi)$. Thus under the condition that $K<\nu$, the system (6.8)-(6.11) has an exponentially tracking random invariant manifold $\mathcal{M}^{\epsilon}(\omega)=\left\{\left(h^{\epsilon}\left(\omega, Y_{0}\right), Y_{0}\right) Y_{0} \in H_{2}\right\}$ when $\epsilon>0$ is sufficiently small. In particular, by Theorem 4.4 the system (6.8)-(6.11) has a reduction equation

$$
\left\{\begin{array}{l}
\dot{\tilde{y}}^{\epsilon}=\mathcal{B} \tilde{y}^{\epsilon}+G\left(\tilde{x}, \tilde{y}^{\epsilon}\right), \\
\tilde{x}=h^{\epsilon}\left(\theta_{t}^{\epsilon} \omega, \tilde{y}^{\epsilon}\right),
\end{array}\right.
$$

where $\mathcal{B}$ and $G$ are defined as in Example 6.1. 


\section{Remarks on the case of local Lipschitz nonlinearity}

We have limited ourselves to the case where the nonlinearities are globally Lipschitz continuous. We remark that when the nonlinearities in those three examples in Section 6 are only locally Lipschitz (say, near the origin $(0,0)$ ), the above discussions remain valid locally. To this end we state the definition of a local random invariant manifold $[4,8]$.

DEFINITION 7.1. We say that the random dynamical system $\phi(t, \omega)$ has a local random invariant manifold (LRIM) with radius $R$ if there is a random set $\mathcal{M}^{R}(\omega)$, which is defined by the graph of a random continuous function $\psi(\omega, \cdot): \overline{B_{R}(0)} \bigcap H_{2} \rightarrow H_{1}$, such that for all bounded sets $B$ in $B_{R}(0) \subset H_{2}$ we have

$$
\phi(t, \omega)\left[\mathcal{M}^{R}(\omega) \bigcap B\right] \subset \mathcal{M}^{R}\left(\theta_{t} \omega\right)
$$

for all $t \in\left(0, \tau_{0}(\omega)\right)$ with

$$
\tau_{0}(\omega)=\tau_{0}(\omega, B)=\inf \left\{t \geq 0: \phi(t, \omega)\left[\mathcal{M}^{R}(\omega) \bigcap B\right] \not \subset B_{R}(0)\right\} .
$$

Let $\chi: H_{1} \times H_{2} \rightarrow \mathbb{R}$ be a bounded smooth function such that

$$
\chi\left(v_{1}, v_{2}\right)= \begin{cases}1, & \text { if }\left\|v_{1}\right\|_{1}+\left\|v_{2}\right\|_{2} \leq 1, \\ 0, & \text { if }\left\|v_{1}\right\|_{1}+\left\|v_{2}\right\|_{2} \geq 2 .\end{cases}
$$

For any positive parameter $R$, we define $\chi_{R}\left(v_{1}, v_{2}\right)=\chi\left(\frac{v_{1}}{R}, \frac{v_{2}}{R}\right)$ for all $\left(v_{1}, v_{2}\right) \in H_{1} \times H_{2}$. Let $f^{(R)}(x, y):=\chi_{R}(x, y) f(x, y), g^{(R)}(x, y):=\chi_{R}(x, y) g(x, y)$. For every $R>0$, there must exist a positive $K_{R}$ such that

$$
\left\|f^{(R)}\left(x_{1}, y_{1}\right)-f^{(R)}\left(x_{2}, y_{2}\right)\right\|_{1} \leq K_{R}\left(\left\|x_{1}-x_{2}\right\|_{1}+\left\|y_{1}-y_{2}\right\|_{2}\right)
$$

and

$$
\left\|g^{(R)}\left(x_{1}, y_{1}\right)-g^{(R)}\left(x_{2}, y_{2}\right)\right\|_{2} \leq K_{R}\left(\left\|x_{1}-x_{2}\right\|_{1}+\left\|y_{1}-y_{2}\right\|_{2}\right) .
$$

Then the cut-off system of (3.4)-(3.5) is as follows:

$$
\begin{aligned}
& d X^{\epsilon}=\frac{1}{\epsilon} A X^{\epsilon} d t+\frac{1}{\epsilon} F^{(R)}\left(X^{\epsilon}, Y^{\epsilon}, \theta_{t}^{\epsilon} \omega\right) d t, \\
& d Y^{\epsilon}=B Y^{\epsilon} d t+G^{(R)}\left(X^{\epsilon}, Y^{\epsilon}, \theta_{t}^{\epsilon} \omega\right) d t
\end{aligned}
$$

where

$$
\begin{aligned}
& F^{(R)}\left(X^{\epsilon}, Y^{\epsilon}, \theta_{t}^{\epsilon} \omega\right)=f^{(R)}\left(X^{\epsilon}+\eta^{\frac{1}{\epsilon}}\left(\theta_{t} \omega\right), Y^{\epsilon}\right), \\
& G^{(R)}\left(X^{\epsilon}, Y^{\epsilon}, \theta_{t}^{\epsilon} \omega\right)=g^{(R)}\left(X^{\epsilon}+\eta^{\frac{1}{\epsilon}}\left(\theta_{t} \omega\right), Y^{\epsilon}\right) .
\end{aligned}
$$

The system (7.1)-(7.2) has a unique solution and thus the solution mapping generates a continuous random dynamical system $\Phi_{R}^{\epsilon}$. If $K_{R}<\gamma_{1}$, then the cut-off system (7.1)(7.2) admits a globally invariant manifold $\mathcal{M}_{R}^{\epsilon}$ possessing the exponentially tracking property. Now as $\Phi^{\epsilon}$ and $\Phi_{R}^{\epsilon}$ agree on $B_{R}(0)$, we conclude that $\widetilde{\mathcal{M}}_{R}^{\epsilon}=\mathcal{M}_{R}^{\epsilon} \cap B_{R}(0)$ defines a local invariant manifold of the original system (3.4)-(3.5).

Acknowledgment. We would like to thank Bjorn Schmalfußfor pointing out this problem to us and thank Wei Wang, Guanggan Chen, and Jicheng Liu for helpful discussions and comments. 


\section{REFERENCES}

[1] L. Arnold, Random Dynamical Systems, Springer-Verlag, Berlin, 1998.

[2] P. Bates, K. Lu, and C. Zeng, Existence and Persistence of Invariant Manifolds for Semiflows in Banach Space, Mem. Amer. Math. Soc., 135, 1998.

[3] A. Bensoussan and F. Flandoli, Stochastic inertial manifold, Stochastics Rep., 53, 13-39, 1995.

[4] D. Blomker and W. Wang, Qualitative properties of local random invariant manifolds for SPDE with quadratic nonlinearity, J. Dyn. Diff. Eqs., 22, 677-695, 2010.

[5] T. Caraballo, P.E. Kloeden, and B. Schmalfuß, Exponentially stable stationary solutions for stochastic evolution equations and their perturbation, Appl. Math. Optim., 50, 183-207, 2004.

[6] T. Caraballo, I. Chueshov, and J.A. Langa, Existence of invariant manifolds for coupled parabolic and hyperbolic stochastic partial differential equations, Nonlin., 18, 747-767, 2005.

[7] C. Castaing and M. Valadier, Convex Analysis and Measurable Multifunctions, LNM 580, Springer-Verlag, Berlin, Heidelberg, New York, 1977.

[8] G. Chen, J. Duan, and J. Zhang, Geometric shape of invariant manifolds for a class of stochastic partial differential equations, J. Math. Phys., 52, 072702, 2011.

[9] C. Chicone and Y. Latushkin, Center manifolds for infinite dimensional nonautonomous differential equations, J. Diff. Eqs., 141, 365-399, 1997.

[10] P.L. Chow, Thermoelastic wave propagation in a random medium and some related problems, Int. J. Eng. Sci., 11, 253-971, 1973.

[11] S.N. Chow, K. Lu, and X.B. Lin, Invariant manifolds for flows in Banach space, J. Diff. Eqs., 74, 285-317, 1988.

[12] S.N. Chow, K. Lu, and X.B. Lin, Smooth foliations for flows in Banach space, J. Diff. Eqs., 94, 266-291, 1991.

[13] J. Cronin, Mathematical Aspects of Hodgkin-Huxley Neural Theory, Cambridge Univ. Press, Cambridge, 1987.

[14] I. Chueshov and B. Schmalfuß, Master-slave synchronization and invariant manifolds for coupled stochastic systems, J. Math. Phys., 51, (102702), 2010.

[15] I. Chueshov, Approximate inertial manifolds of exponential order for semilinear parabolic equations subjected to additive white noise, J. Dynam. Diff. Eqs., 7, 549-566, 1995.

[16] I. Chueshov, A reduction principle for coupled nonlinear parabolic-hyperbolic PDEs, J. Evo. Eqs., 4, 591-612, 2004.

[17] J. Duan, K. Lu, and B. Schmalfuß, Invariant manifolds for stochastic partial differential equations, Ann. Probab., 31, 2109-2135, 2003.

[18] J. Duan, K. Lu, and B. Schmalfuß, Smooth stable and unstable manifolds for stochastic evolutionary equations, J. Dynam. Diff. Eqs., 16, 949-972, 2004.

[19] R. Fitzhugh, Impulse and physiological states in models of nerve membrane, Biophys. J., 1, 445-466, 1961.

[20] J. Hadamard, Sur l'iteration et les solutions asymptotiques des equations differentielles, Bull. Soc. Math. France, 29, 224-228, 1901.

[21] D. Henry, Geometric Theory of Semilinear Parabolic Equations, Springer-Verlag, Berlin, 1981.

[22] R.G. Littlejohn and W.G. Flynn, Geometric phases in the asymptotic theory of coupled wave equations, Phys. Rev. A, 44, 5239, 1991.

[23] K. Lu and B. Schmalfuß, Invariant foliations for stochastic partial differential equations, Stoch. Dyn., 8, 505-518, 2008.

[24] A.M. Lyapunov, Problème géneral de la stabilité du mouvement, Ann. of Math. Stud., 17, 1947 (originally published in Russian, 1892).

[25] S.E.A. Mohammed, T. Zhang, and H. Zhao, The stable manifold theorem for semilinear stochastic evolution equations and stochastic partial differential equations, Mem. Amer. Math. Soc., 196, 1-105, 2008.

[26] M. Najafi, G.R. Sarhangi, and H. Wang, Stabilizability of coupled wave equations in parallel under various boundary conditions, IEEE Trans. Auto. Control, 42, 1308-1312, 1997.

[27] J.S. Nagumo, S. Arimoto, and S. Yoshizawa, An active pulse transmission line simulating nerve axon, Proc. IRE, 50, 2061-2071, 1962.

[28] A. Pazy, Semigroups of Linear Operators and Applications to Partial Differential Equations, Springer-Verlag, Berlin, 1983.

[29] O. Perron, Über stabilität und asymptotisches verhalten der integrale von differentialgleichungssystemen, Math. Z, 29, 129-60, 1928.

[30] D. Ruelle, Characteristic exponents and invariant manifolds in Hilbert space, Ann. Math., 115, 243-290, 1982.

[31] B. Schmalfuß, A random fixed point theorem and random graph transformation, J. Math. Anal. 
Appl., 225, 91-113, 1998 .

[32] B. Schmalfuß and R. Schneider, Invariant manifolds for random dynamical systems with slow and fast variables, J. Dynam. Diff. Eqs., 20, 133-164, 2008.

[33] K. Yosida, Functional Analysis, Springer-Verlag, New york, 1968.

[34] W. Wang and A.J. Roberts, Slow manifold and averaging for slow-fast stochastic differential system, http://arxiv.org/abs/0903.1375. 